\title{
Acknowledgement to Reviewers of Journal of Sensor and Actuator Networks in 2019
}

\author{
Journal of Sensor and Actuator Networks Editorial Office \\ MDPI, St. Alban-Anlage 66, 4052 Basel, Switzerland
}

Published: 16 January 2020

\begin{abstract}
The editorial team greatly appreciates the reviewers who have dedicated their considerable time and expertise to the journal's rigorous editorial process over the past 12 months, regardless of whether the papers are finally published or not. In 2019, a total of 56 papers were published in the journal, with a median time to first decision of 17 days and a median time from submission to publication of 46 days. The editors would like to express their sincere gratitude to the following reviewers for their generous contribution in 2019:
\end{abstract}

\begin{tabular}{|c|c|}
\hline Abdullah, Oday Ibraheem & De La Prieta Pintado, Fernando \\
\hline Albuquerque, Daniel & Del Peral-Rosado, José A. \\
\hline Alejos, Ana Vázquez & Deng, Shuo \\
\hline Al-Hadhrami, Tawfik & Díez-González, Javier \\
\hline Aloqaily, Moayad & Dinh, Anh \\
\hline Amanowicz, Marek & Dombek, Grzegorz \\
\hline Amaro, José Pedro & Dragan, Ioan \\
\hline Anagnostopoulos, Marios & Dreżewski, Rafał \\
\hline Anjangi, Prasad & Dumbrava, Stefan \\
\hline Arjoune, Youness & Duroc, Yvan \\
\hline Arriola, Aitor & Estrada-López, Johan J. \\
\hline Bahsi, Hayretdin & Fernandez-Veiga, Manuel \\
\hline Barreto, Luís Manuel C. & Feuz, Kyle D. \\
\hline Bauer, Jan & Fiala, Pavel \\
\hline Bellekens, Ben & Fisher, Daniel K. \\
\hline Benzerrouk, Hamza & Franco Silva, Edelberto \\
\hline Blech, Jan Olaf & Fung, W.K. \\
\hline Boada, Maria Jesus Lopez & G Menon, Varun \\
\hline Borhani, Alireza & Garcia Gonçalves, Luiz Marcos \\
\hline Bradbury, Matthew & Ghoreyshi, Seyed Mohammad \\
\hline Cecílio, José & Ghosh, Monisha \\
\hline Charvatova, Hana & Giannelli, Carlo \\
\hline Chen, Feng & Goga, Nicolae \\
\hline Chen, Liang-Bi & Goleva, Rossitza \\
\hline Chen, Lien-Wu & Gomez Blas, Nuria \\
\hline Chen, Yuan-Ho & Gonzalez Crespo, Ruben \\
\hline Choi, Wooyeol & Gonzalez, Hugo \\
\hline Ciuonzo, Domenico & González-Briones, Alfonso \\
\hline Comisso, Massimiliano & Gregory, Mark \\
\hline Corchia, Laura & Grossi, Marco \\
\hline Correia, Sérgio & Guerra, Jorge \\
\hline Cosentino, Valentina & Harik, El Houssein Chouaib \\
\hline Covaci, Stefan & Hasanian, Mostafa \\
\hline Cui, Zheng & Hernández-Ramos, José L. \\
\hline Curiac, Daniel-Ioan & Huang, Tao \\
\hline Daamen, Winnie & Ishihara, Susumu \\
\hline Damasevicius, Robertas & Kaabouch, Naima \\
\hline Danel, Roman & Kadel, Rajan \\
\hline
\end{tabular}


Kakarountas, Athanasios

Kargas, Georgios

Karimov, Timur

Karkazis, Panagiotis A.

Kathiravelu, Pradeeban

Ke, ChihKun

Kelner, Jan

Kenawy, Hamed Salah

Khan, Jamil Y.

Khan, Pervez

Khooban, Mohammad Hassan

Kim, Taehong

Kolomvatsos, Kostas

Kong, Kian Hau

Korbel, Piotr

Kourtis, Michael-Alexandros

Krems, Josef F.

Kutzner, Tobias

Kwon, Minhae

Lang, Walter

Lee, Il-Gu

Lee, Jae Woong

Lee, Sang Hyun

Lee, Yangsun

Li, Ying

Li, Yundong

Liang, Jing

Lin, Shih-Chun

Liu, Anfeng

Liu, Jiechao

Liu, Lianggui

Liu, Wei-Cheng

Lloret, Jaime

Lupu, Ciprian

Luque, Joaquín

Luque-Nieto, Miguel Angel

Macko, Dominik

Madurapperuma, Buddhika

Maiti, Ananda

Marco Gisbert, Hector

Masini, Barbara Mavì

Massaroni, Carlo

Mendicute, Mikel

Meng, Weizhi

Mikhaylov, Konstantin

Møgelmose, Andreas

Moreno-Guerrero, Antonio-José

MURASE, Tutomu

Muthanna, Ammar

Namiot, Dmitry

Newe, Thomas

Nguyen, Van Dung

Oberweger, Markus

Ojo, Mike

O'Mahony, Donal

Omar, Abbas

Orosz, György

Orue, Amalia
Otebolaku, Abayomi

Pace, Pasquale

Palos-Sánchez, Pedro

Panda, Manoj

Paolo, Favali

Papageorgas, Panagiotis

Parada Medina, Raúl

Patryk, Kot

Pérez de Prado, Rocío Josefina

Picone, Marco

Płaczek, Bartłomiej

Poniszewska-Maranda, Aneta

Pozzebon, Alessandro

Prasad, Mukesh

$\mathrm{Pu}$, Cong

Qiu, Han

Ramírez-Faz, Jose

Ramos, Victor

Raz, Ali

Reis, Manuel J.C.S.

Rezapour, Mahdi

Righi, Rodrigo

Rizzardi, Alessandra

Rodrigues, Thiago Braga

Roscia, Mariacristina

Rossi, Massimiliano

Rotariu, Cristian

Rydosz, Artur

Salam, Adul

Sánchez, Borja Bordel

Sassani, Alireza

Schmitt, Corinna

Shanmugam, Bharanidharan

Shen, Haoting

Shi, Yong

Shibata, Naoki

Silvani, Xavier

Soběslav, Vladimír

Soltanpur, Cinna

Sood, Keshav

Sousa, Armando Jorge

Spanakis, Emmanouil G.

Stateczny, Andrzej Eugeniusz

Suciu, George

Šumak, Boštjan

Svecko, Rajko

Szeidert, Iosif

Szkaliczki, Tibor

Taheri, Pooya

Tamberg, Gert

Tomasz, Rymarczyk

Torres-Sospedra, Joaquín

Tortia, Cristina

Trakadas, Panagiotis

Tsang, Kim Fung

Valente, Antonio

Varga, Pal

Verde, Francesco 
Villadangos, Jesus Vulpe, Alexandu

Xia, Minghua

Xiong, Wen

Yelamarthi, Kumar

Yeom, Junghoon

Yin, Zhendong
Yu, Yang

Yue, Jing

Zabierowski, Wojciech

Zabini, Flavio

Zavadskas, Edmundas Kazimieras

Zhang, Qing

Zikria, Yousaf Bin

(C) 2020 by the authors. Licensee MDPI, Basel, Switzerland. This article is an open access article distributed under the terms and conditions of the Creative Commons Attribution (CC BY) license (http://creativecommons.org/licenses/by/4.0/). 